\title{
Dynamics of a stochastic phytoplankton-toxic phytoplankton-zooplankton system under regime switching
}

\author{
He Liu ${ }^{1}$, Chuanjun Dai ${ }^{1}$, Hengguo $\mathrm{Yu}^{2}$, Qing Guo ${ }^{1}$, Jianbing $\mathrm{Li}^{3}$, Aimin Hao ${ }^{1}$, Jun \\ $\mathrm{Kikuchi}^{4}$, and Min Zhao ${ }^{5}$ \\ ${ }^{1}$ Wenzhou University \\ ${ }^{2}$ School of Mathematical and Electronic Information Engineering, Wenzhou University, \\ Wenzhou, Zhejiang, 325035, CHINA \\ ${ }^{3}$ University of Northern British Columbia \\ ${ }^{4}$ Environmental Metabolic Analysis Research Team, RIKEN Center for Sustainable \\ Resource Science \\ ${ }^{5}$ School of Life and Environmental Science
}

February 12, 2021

\begin{abstract}
In this paper, a stochastic phytoplankton-toxic phytoplankton-zooplankton system with Beddington-DeAngelis functional response, where both the white noise and regime switching are taken into account, is studied analytically and numerically. The aim of this research is to study the combined effects of the white noise, regime switching and toxin-producing phytoplankton (TPP) on the dynamics of the system. Firstly, the existence and uniqueness of global positive solution of the system is investigated. Then some sufficient conditions for the extinction, persistence in the mean and the existence of a unique ergidoc stationary distribution of the system are derived. Significantly, some numerical simulations are carried to verify our analytical results, and show that high intensity of white noise is harmful to the survival of plankton populations, but regime switching can balance the different survival states of plankton populations and decrease the risk of extinction. Additionally, it is found that an increase in the toxin liberation rate produced by TPP will increase the survival change of phytoplankton, while it will reduce the biomass of zooplankton. All these results may provide some insightful understanding on the dynamics of phytoplankton-zooplankton system in randomly disturbed aquatic environments.
\end{abstract}

\section{Hosted file}

Dynamics of a stochastic phytoplankton-toxic phytoplankton-zooplankton system under regime switching.pd available at https://authorea.com/users/395345/articles/508643-dynamics-of-a-stochasticphytoplankton-toxic-phytoplankton-zooplankton-system-under-regime-switching 